\title{
Management of Biliopancreatic Limb Bleeding after Roux-en-Y Gastric Bypass: A Case Report
}

\author{
Christophe Riquoir ${ }^{1 *}$, Luis Antonio Díaz ${ }^{2 *}$, David Chiliquinga ${ }^{3}$, Roberto Candia ${ }^{2}$, Fernando Pimentel ${ }^{4}$ and Alex Arenas ${ }^{2,5}$ \\ ${ }^{1}$ School of Medicine, Pontificia Universidad Católica de Chile, ${ }^{2}$ Department of Gastroenterology, School of Medicine, Pontificia \\ Universidad Católica de Chile, ${ }^{3}$ Department of Internal Medicine, School of Medicine, Pontificia Universidad Católica de Chile, \\ ${ }^{4}$ Department of Digestive Surgery, Division of Surgery, School of Medicine, Pontificia Universidad Católica de Chile, ${ }^{5}$ Unit of \\ Gastroenterology, Complejo Asistencial Dr. Sótero Del Río, Santiago, Chile
}

The Roux-en-Y gastric bypass is one of the most extensive surgical treatments for obesity. The treatment of upper gastrointestinal bleeding after Roux-en-Y gastric bypass is complex due to the difficulty of accessing the excluded gastric antrum and duodenal bulb. There is no consensus regarding the management of this complication. While various techniques have been described to access the biliopancreatic limb, double-balloon enteroscopy is the most commonly used. If double-balloon enteroscopy is unavailable, a pediatric colonoscope may be used as an alternative; however, its use in such cases has not been described. We report the case of a 50 -year-old male patient who underwent gastric bypass 13 years ago and was admitted for a second episode of upper gastrointestinal bleeding. The initial approach using upper endoscopy, colonoscopy, and abdominal computed tomography angiography did not reveal the cause of gastrointestinal hemorrhage; therefore, an endoscopic study of the biliopancreatic limb was performed using a pediatric colonoscope. A Forrest Ib ulcer was found in the duodenal bulb, and endoscopic therapy was administered. The evolution was found to be satisfactory. Clin Endosc 2021;54:754-758

Key Words: Colonoscope; Double-Balloon Enteroscopy; Duodenal Ulcer; Gastric Bypass; Gastrointestinal Hemorrhage

\section{INTRODUCTION}

In $2016,39 \%$ of the world's population was overweight and $13 \%$ were obese. ${ }^{1}$ Roux-en-Y gastric bypass (RYGB) is frequently used as a surgical treatment for obesity and its comorbidities. Although highly effective, this procedure is not exempt from complications, which occur in approximately $20 \%$ of cases. ${ }^{2}$ The most frequent complications are stenosis (8\%-19\%), gastro-jejunal or jejunum-jejunal anastomosis

Received: January 25, 2021 Revised: March 8, 2021

Accepted: March 15, 2021

Correspondence: Alex Arenas

Gastroenterology \& Hepatology, Pontificia Universidad Católica de Chile, Diagonal Paraguay 362, Santiago, Chile

Tel: +56-9-982563166, Fax: +56-2-23543850, E-mail: afarenas@gmail.com ORCID: https://orcid.org/0000-0002-0901-966X

*These authors contributed equally to this study.

cc This is an Open Access article distributed under the terms of the Creative Commons Attribution Non-Commercial License (http://creativecommons.org/ licenses/by-nc/3.0) which permits unrestricted non-commercial use, distribution, and reproduction in any medium, provided the original work is properly cited. leakage (0.6\%-4.4\%), and thromboembolic disease (3\%). Gastrointestinal hemorrhage accounts for up to $11 \%$ of early complications and commonly originates in the gastrojejunal anastomosis suture line. However, this can also occur in the jejunum-jejunal anastomosis or in the excluded limb, including the gastric remnant and duodenum. Late complications, although less common, include marginal ulcers (2\%-5\%), perforation (1\%-2\%), hernias, and bowel obstruction. Gastrointestinal bleeding is very infrequent in the late postoperative period. ${ }^{3}$ We present the case of a patient with a clinical history of RYGB admitted for upper gastrointestinal bleeding, and we will discuss the therapeutic approach in this scenario.

\section{CASE REPORT}

A 50-year-old man with obesity who underwent RYGB 13 years ago presented to the emergency department with four days of melena, abdominal distension, and progressive dyspnea. He self-medicated with ibuprofen $600 \mathrm{mg} /$ day for the 

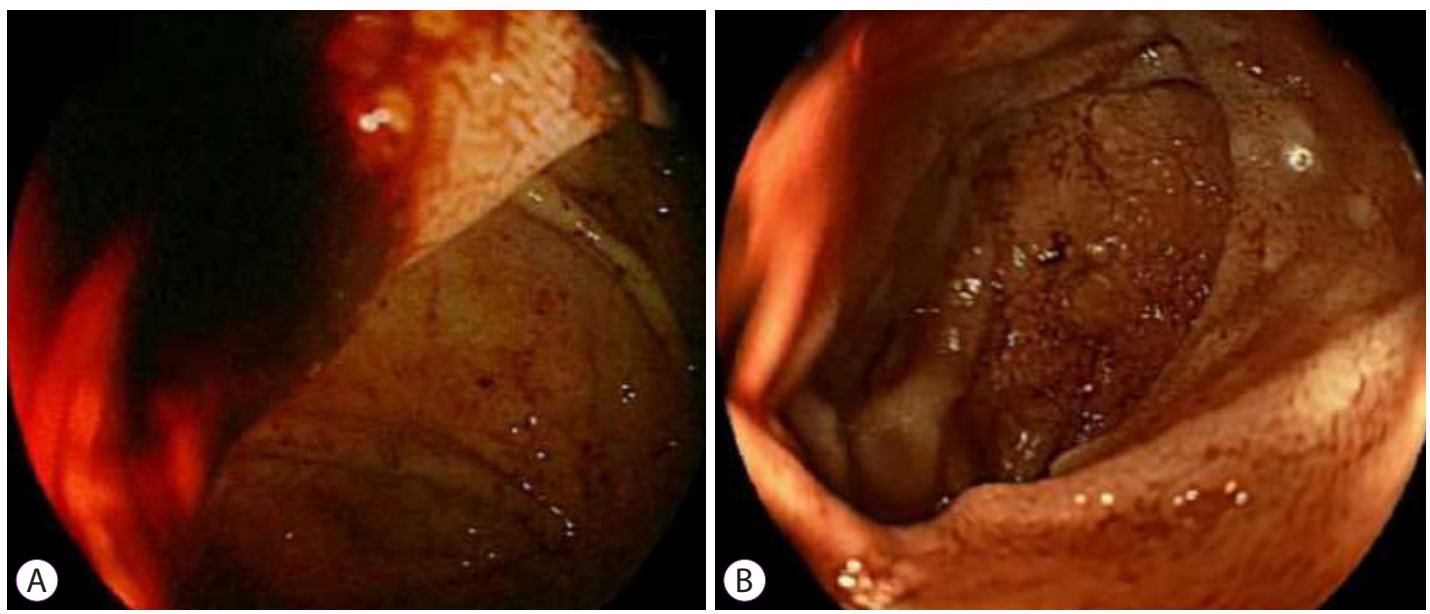

Fig. 1. Colonoscopic findings. (A) Fresh blood from the ileum (ileocecal valve), suggesting an upper origin of the bleeding. (B) lleum with presence of fresh blood.
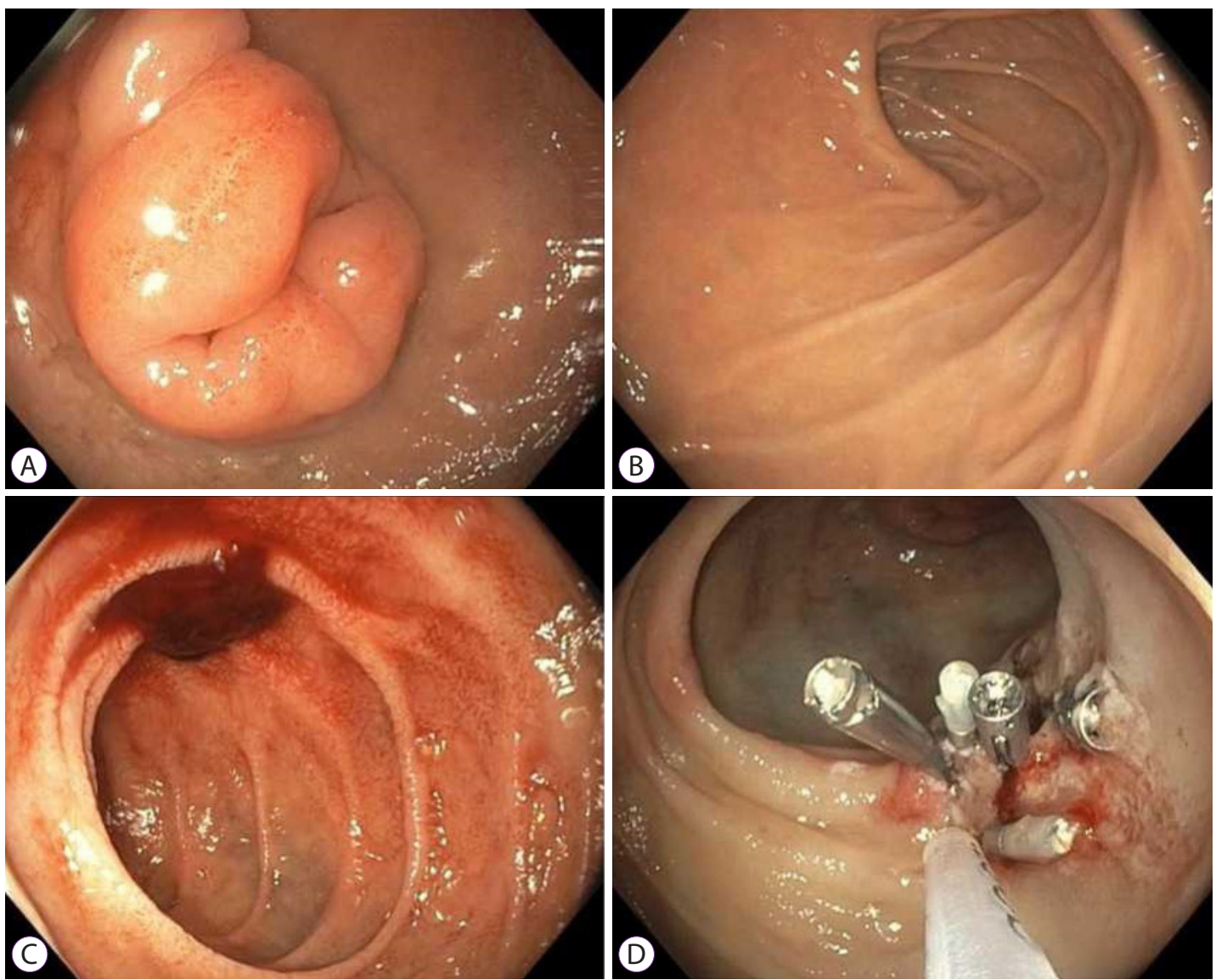

Fig. 2. Enteroscopy with a pediatric colonoscope through the biliopancreatic limb. (A) Identification of the pylorus with erosions (view from the duodenum). (B) View of the gastric greater curvature from the antrum. (C) An ulcer was detected in the proximal duodenal region (posterior wall) with oozing bleeding. (D) Injection therapy with adrenaline, thermocoagulation with a bipolar probe, and four hemoclips, of which three were effective. Thereafter, hemostasis was completed with hemospray. 
last three days. He noted occasional alcohol consumption but denied smoking. He was hospitalized five years ago for gastrointestinal hemorrhage and, at that time, presented with similar clinical manifestations but without nonsteroidal anti-inflammatory drug (NSAID) use. During his previous hospitalization, upper and lower endoscopic examinations did not reveal any lesions. At the emergency department, the patient was hemodynamically stable and physical examination did not reveal any pertinent findings. High-dose intravenous omeprazole was administered and upper endoscopy was performed; however, no evidence of recent bleeding was found, and the gastrojejunal anastomosis was permeable and had no lesions. The study was complemented by colonoscopy. After ingesting a laxative solution, the patient was noted to have hematochezia and hemodynamic instability. Laboratory tests confirmed a significant decrease in hemoglobin level (from $10 \mathrm{~g} / \mathrm{dL}$ to 7.5 $\mathrm{g} / \mathrm{dL}$ ), and two units of red blood cells were transfused.

Colonoscopy did not reveal any lesions, but fresh blood from the ileum was identified (Fig. 1). An abdominopelvic computed tomography angiography (CTA) was subsequently performed; however, the site of bleeding still could not be identified. Given the recent use of NSAIDs, an upper endoscopy using a pediatric colonoscope was performed to assess the gastric remnant and the duodenum (Fig. 2A, B). After ascending through the biliopancreatic limb, a Forrest Ib ulcer was identified in the duodenal bulb immediately distal to the pylorus (Fig. 2C). Hemostasis was performed using injection therapy, thermocoagulation, and four clips (Fig. 2D). Due to the persistence of oozing bleeding, a hemospray was used to achieve satisfactory hemostasis.

After 72 hours of high-dose omeprazole, monitoring, and no evidence of recurrent bleeding, the patient was discharged. Over 8-months of follow-up consults, the patient remained asymptomatic and without any new hemorrhages.

\section{DISCUSSION}

Gastrointestinal bleeding following RYGB has an incidence rate of $0.4 \%-4.4 \%$ and can occur in either the early ( $<30$ days) or late ( $>30$ days) postoperative period, with each having different causes and approaches. Early events are usually associated with anastomotic leaks and hemorrhage in the staple line, most frequently in the gastrojejunostomy and jejunojejunostomy, with the latter usually manifesting as intra-abdominal bleeding or hemoperitoneum. Conservative management for these complications is often sufficient; however, in unstable patients or those that require repeated transfusion, surgery was almost always the treatment of choice in the past until an endoscopic approach proved to be a safe and equally effective alternative in selected cases. ${ }^{4,5}$

Late gastrointestinal bleeding is rare and can present as melena/hematochezia, chronic bleeding with iron deficiency anemia, or heme-positive stools. Marginal ulcers in anastomosis sites are the most common cause of late bleeding in bypassed patients. ${ }^{6}$ In addition, a tense anastomosis favors microvascular ischemia and neovascularization, which increases the risk of bleeding. Peptic ulcers in the gastric remnant can also be a late cause of bleeding as these patients are susceptible to several risk factors for peptic disease, such as Helicobacter pylori ( $H$. pylori) infection, use of NSAIDs, smoking, and preserved acid secretion in the gastric pouch. Additionally, the occasional passage of non-absorbable sutures into the lumen favors the formation of ulcers. ${ }^{4,6}$

These events constitute a complex scenario especially when upper and lower endoscopic examinations do not identify the source of bleeding. Bleeding from the excluded segments is a rare phenomenon, and while there are no specific clinical guidelines available, several management algorithms have been proposed. ${ }^{7}$ To date, there is no consensus about the endoscopic approach.

Generally, the patient's clinical presentation can provide clues to the source of bleeding; hematemesis suggests a proximal source (the gastric pouch and/or the gastrojejunal anastomosis) and can be quickly excluded with an upper endoscopy. Upper endoscopy is frequently used only in late postoperative bleeding due to the high risk of dehiscence and perforation associated with its use in the early postoperative period. Therefore, upper endoscopy is only considered in cases of rebleeding after conservative management or in patients who present with hemodynamic instability.

In cases of gastrointestinal bleeding following RYGB without evident lesions on upper endoscopy, subsequent decisions will depend on the patient's stability and clinical suspicion. In hemodynamically stable patients, a lower origin of bleeding can be ruled-out using colonoscopy. ${ }^{8}$ An imaging study with contrast, such as CTA, is also useful especially when active bleeding is suspected. In this case, colonoscopy showed blood and blood clots coming from the ileum, therefore increasing the suspicion of an upper source of the bleeding. If CTA does not reveal the bleeding site, the next step is to study the biliopancreatic limb.

There are several alternatives to explore the excluded limb, including double-balloon enteroscopy (DBE), mono balloon enteroscopy, push enteroscopy, and intraoperative laparoscopic endoscopy. Currently, DBE is recommended by the American Society for Gastrointestinal Endoscopy for studying occult bleeding in small bowels with altered anatomy; DBE 
has proven to be useful in RYGB, even for the evaluation of the excluded stomach and duodenum. ${ }^{9}$ A second upper endoscopy may enable access to the biliary limb but is unlikely to reach the duodenum and gastric remnant. An endoscopic capsule is not recommended as it cannot access the excluded limb.

In the absence of DBE, an expert endoscopist usually modifies the technique or instrument to access difficult segments. Push enteroscopy with a colonoscope has proven to be useful in these scenarios, ${ }^{10}$ although its utility has not been compared with DBE. In this case, the use of a pediatric colonoscope allowed passage through the narrow and angled course to the excluded limb (Fig. 3). After the lesion was identified, the wide working channel of the colonoscope facilitated satisfactory endoscopic therapy with traditional devices. ${ }^{8}$ This is one advantage over DBE, which has a narrow working channel that limits its compatibility with traditional devices. However, the length of the biliopancreatic limb is an important factor in the success of the procedure. RYGB surgery was usually performed with a long biliopancreatic limb length which is associated with malnutrition and liver dysfunction in some patients. ${ }^{11}$ To avoid these complications, the biliopancreatic limb length has been shortened. This difference could explain why previous articles could not reach the duodenum using a

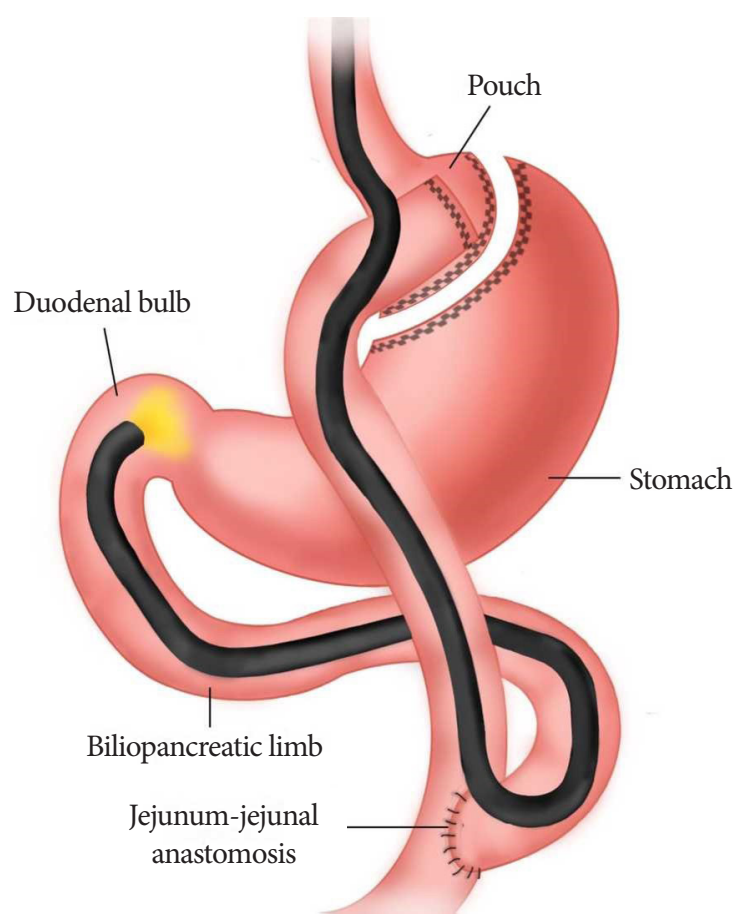

Fig. 3. Passage of the pediatric colonoscope through the alimentary limb with return through the anastomosis of the excluded limb. pediatric colonoscope. ${ }^{12,13}$ If the source of the bleeding is not identified by endoscopy, an alternative approach is to use angiography with interventional radiology. ${ }^{8}$ This strategy is preferred in surgically high-risk patients; however, because of the terminal irrigation of the jejunum and ileum, there is a higher risk of developing segmental necrosis following embolization. Furthermore, there is also a risk of devascularization of the gastric pouch, which depends on the left gastric artery. Nevertheless, current techniques enable selective embolization to decrease this risk. When the above alternatives do not stop the bleeding, surgery is needed. Because revision surgery in the acute period is associated with higher morbidity and mortality $(10 \%-30 \%$ versus $<2 \%$ in elective surgery), stabilization up to a semi-elective condition is recommended. ${ }^{6,8}$

Approximately $60 \%$ of cases of bleeding recurrence occur in the same lesion. ${ }^{14}$ In this case, even if the origin of the bleeding in the first event was not found, it was likely the same as that in the second event. Prevention of peptic rebleeding is based on modifying risk factors such as smoking, use of NSAIDs or antiplatelet drugs, and detection and eradication of $\mathrm{H}$. pylori. ${ }^{15}$ Proton-pump inhibitors reduce the risk of delayed bleeding secondary to peptic disease in the gastric remnant and duodenum. However, while its prophylactic use after RYGB has been proposed, its potential adverse effects are controversial. ${ }^{6}$

Gastrointestinal bleeding from the excluded limb in bypassed patients is a challenge for endoscopists and surgeons. After upper endoscopy, colonoscopy, and CTA without findings, push enteroscopy using a pediatric colonoscope could be considered in the study of a biliopancreatic limb. Prospective studies are needed to compare this strategy to other endoscopic alternatives.

Conflicts of Interest

The authors have no potential conflicts of interest.

Funding

None.

Acknowledgments

We gratefully acknowledge the team of the endoscopy unit of the Red de Salud UC CHRISTUS.

ORCID
Christophe Riquoir A: Luis Antonio Díaz: David Chiliquinga M.: Roberto Candia: Fernando Pimentel: Alex Arenas: https://orcid.org/0000-0001-7462-2708 https://orcid.org/0000-0002-8540-4930 https://orcid.org/0000-0003-3293-0731 https://orcid.org/0000-0003-1856-7737 https://orcid.org/0000-0001-5891-1008 https://orcid.org/0000-0002-0901-966X 


\section{REFERENCES}

1. World Health Organization. Obesity and overweight [Internet]. Geneva: WHO; c2020 [updated 2020 Apr 1; cited 2021 Apr 2]. Available from: https://www.who.int/news-room/fact-sheets/detail/obesity-and-overweight

2. Coblijn UK, Karres J, de Raaff CAL, et al. Predicting postoperative complications after bariatric surgery: the Bariatric Surgery Index for Complications, BASIC. Surg Endosc 2017;31:4438-4445.

3. Hendrickson CM, Gibb SL, Miyazawa BY, et al. Elevated plasma levels of TIMP-3 are associated with a higher risk of acute respiratory distress syndrome and death following severe isolated traumatic brain injury. Trauma Surg Acute Care Open 2018;3:e000171.

4. Fernández-Esparrach G, Bordas JM, Pellisé M, et al. Endoscopic management of early GI hemorrhage after laparoscopic gastric bypass. Gastrointest Endosc 2008;67:552-555.

5. Heneghan HM, Meron-Eldar S, Yenumula P, Rogula T, Brethauer SA Schauer PR. Incidence and management of bleeding complications after gastric bypass surgery in the morbidly obese. Surg Obes Relat Dis 2012;8:729-735.

6. Coblijn UK, Lagarde SM, Tuynman JB, van Meyel JJM, van Wagensveld BA. Delayed massive bleeding two years after Roux-en-Y gastric bypass. JSLS 2013;17:476-480.

7. García-García ML, Martín-Lorenzo JG, Torralba-Martínez JA, et al. Emergency endoscopy for gastrointestinal bleeding after bariatric surgery. therapeutic algorithm. Cir Esp 2015;93:97-104.
8. Gralnek IM, Dumonceau J-M, Kuipers EJ, et al. Diagnosis and management of nonvariceal upper gastrointestinal hemorrhage: European Society of Gastrointestinal Endoscopy (ESGE) guideline. Endoscopy 2015;47:a1-a46.

9. Skinner M, Peter S, Wilcox CM, Mönkemüller K. Diagnostic and therapeutic utility of double-balloon enteroscopy for obscure GI bleeding in patients with surgically altered upper GI anatomy. Gastrointest Endosc 2014;80:181-186

10. Ali R, Wild D, Shieh F, et al. Deep enteroscopy with a conventional colonoscope: initial multicenter study by using a through-the-scope balloon catheter system. Gastrointest Endosc 2015;82:855-860.

11. Mahawar KK. Liver Dysfunction with both Roux-en-Y and one-anastomosis gastric bypass is almost exclusively seen with longer than standard limb lengths. Obes Surg 2018;28:548-549.

12. Issa $\mathrm{H}, \mathrm{Al}$-Saif $\mathrm{O}$, Al-Momen $\mathrm{S}$, Bseiso $\mathrm{B}, \mathrm{Al}$-Salem A. Bleeding duodenal ulcer after Roux-en-Y gastric bypass surgery: the value of laparoscopic gastroduodenoscopy. Ann Saudi Med 2010;30:67-69.

13. Zerey M, Sigmon LB, Kuwada TS, Heniford BT, Sing RF. Bleeding duodenal ulcer after roux-en-Y gastric bypass surgery. J Am Osteopath Assoc 2008;108:25-27.

14. Palmer ED. The vigorous diagnostic approach to upper-gastrointestinal tract hemorrhage. A 23-year prospective study of 14,000 patients. JAMA 1969;207:1477-1480

15. Seo JH, Hong SJ, Kim J-H, et al. Long-term recurrence rates of peptic ulcers without Helicobacter pylori. Gut Liver 2016;10:719-725. 